\title{
Descriptive Epidemiology of Malignant primary Osteosarcoma in Tunisia 1980-2016
}

\author{
Feryel Letaief Ksontini, Fatma Guermazi, Meddeb Khadija, Mokrani Amina, \\ Yahiaoui Yosra, Gabsi Azza, Rais Henda, Chraiet Nesrine, Ayadi Mouna, Mezlini \\ Amel
}

Departement of Medical Oncology, Salah Azaiez Institute, Tunis 1006, Tunisia.

\begin{abstract}
Background: Osteosarcoma is a rare malignant tumor of the mesenchymal tissue that accounts for $5 \%$ of all malignant tumors in children and teenager. The purpose of our study was to describe the epidemiological and evolutionary characteristics of osteosarcoma in Tunisia. Methods: It's a retrospective study performed in the Oncology Department of Salah Azaiez Institute from January 1980 to December 2016. We analyzed records of 200 cases of patients with primary high-grade osteosarcoma. Results: The mean incidence was 6 cases per year. The mean age at the time of diagnosis was 19 years with a slight male predominance (55\%). The distribution had a single peak during adolescence and a plateau during adulthood. The average consultation period was 3.4 months. The most common reasons for consultation were the pain (82\%), followed by swelling (74\%), trauma (10\%), fracture $(6.5 \%)$, and functional impairment $(5.5 \%)$. Mean tumor size was $11 \mathrm{~cm}$. Preferred site was long bones in young patients and flat bones in elderly ones. Common osteosarcoma was the most frequent (85\%). Overall survival and progression-free survival at 5 years were respectively $78 \%$ and $90.5 \%$. Conclusion: In summary, this study is a large national population-based incidence report of osteosarcoma in Tunisia.
\end{abstract}

Keywords: Osteosarcome- epidemiology- Tunisia

Asian Pac J Cancer Care, 3 (4), 81-86

\section{Introduction}

Primary malignant bone tumors are rare tumors, accounting for $0.2 \%$ of all cancers [1]. Among these malignancies, osteosarcoma (OS) is the main malignant bone tumor in children that accounts for $35 \%$ of all primary bone malignancies [2]. It has a bimodal distribution with two peaks of incidence [3]. This tumor mainly affects boys during the second decade of life. It has the particularity to sit in the metaphysis of the long bones. However, in the elderly, osteosarcoma is more likely to occur in the axial skeleton locations but sometimes it can be radiation-induced or arises in Paget's disease [4]. Despite its low incidence, OS remains one of the most aggressive humans [5]. Multimodal management has resulted in improved results that have led to an increase in survival rates.

Currently, there is a paucity of information about osteosarcoma in Tunisia. So we performed a retrospective
Submission Date: 08/11/2018Ａcceptance Date: 10/13/2018

study in which epidemiological data pertaining to patient demographics, tumor location, and histological diagnosis were recorded and analyzed.

\section{Materials and Methods}

This retrospective descriptive study was performed in the oncology department of Salah Azaiez Institute over a period of 37 years, from 1980 to 2016 and recorded 200 cases of patients with primary high-grade osteosarcoma. Patient charts were reviewed and data extracted. Parameters studied were the age, sex, discovery circumstances, consultation time, physical signs, radiological signs, histological type, therapeutic modalities (chemotherapy, surgery, radiotherapy) and evolution. All patients underwent a pathological examination by the criteria established by the World Health Organization, which confirmed in all cases the diagnosis of primary high-grade osteosarcoma.

\footnotetext{
Corresponding Author:

Dr. Feryel Letaief Ksontini

Departement of Medical Oncology, Salah Azaiez Institute, Tunis 1006, Tunisia

Email: Feryel.Ksontini@gmail.com
} 
For the classification, we used the International Classification of Diseases for Oncology (ICD-O-3) [6]. We included all malignant primary osteosarcomas (site code C40.0-40.9 and C41.0 -41.9 and histology codes 9180 / 3-9187 / 3, 9192 / 3-9195 / 3, 9200/3) [6]. The group diagnosed before 1999 was classified according to the first edition of (ICD-0). Between 1999 and 2000, diagnosed osteosarcoma cases were initially classified according to the second edition of ICD-O (ICD-O-2) and then converted to the third edition of ICD-O (ICD-O -3) [6-7]. From 2001, the third edition (ICD-03) was used from the outset. Survival data were studied Kaplan Meier method. The search for prognostic factors was performed in a single factor analysis by comparing the survival curves by the Log-Rank test. The data was processed on the $<$ SPSS $18>$ software. A confidence interval of $95 \%$ was considered with a level of significance set at $p<0.05$.

\section{Source of Funding}

No funding source did play a role in this investigation.

\section{Results}

\section{Incidence}

The mean incidence was 6 cases per year. During the 36 years, the incidence of OS showed an increase from 5 cases per year between 1978 and 1993 to 5.2 cases per year between 2005 and 2016. The highest incidence was observed between 1994 and 2004 with a rate of 6.2 cases per year.

We found an 8-year old patient with a family history of retinoblastoma who had a femoral osteosarcoma and an orbital retinoblastoma which were associated with the dysfunction of the tumor suppressor protein RB1.

Age

The mean age at the time of diagnosis was 19 years (range, 2-83 years) for male, 17 years (range, 1-70 years) for female, and 18 years (range, 1-83 years) overall. The distribution of osteosarcoma throughout all ages failed to show a bimodal age distribution and only had a single peak during adolescence and a plateau during adulthood (Figure 1).

Sexes

The male-to-female ratio of 1,2 . This predominance

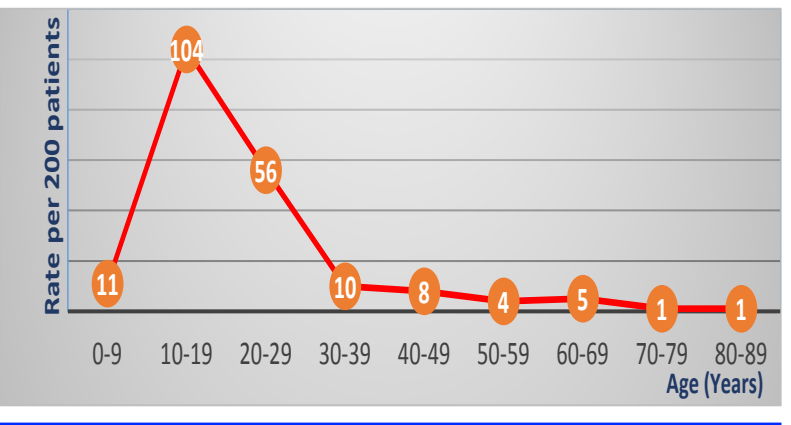

Figure 1. Age Distribution, A Single Peak During Adolescence and a Plateau During Adulthood.

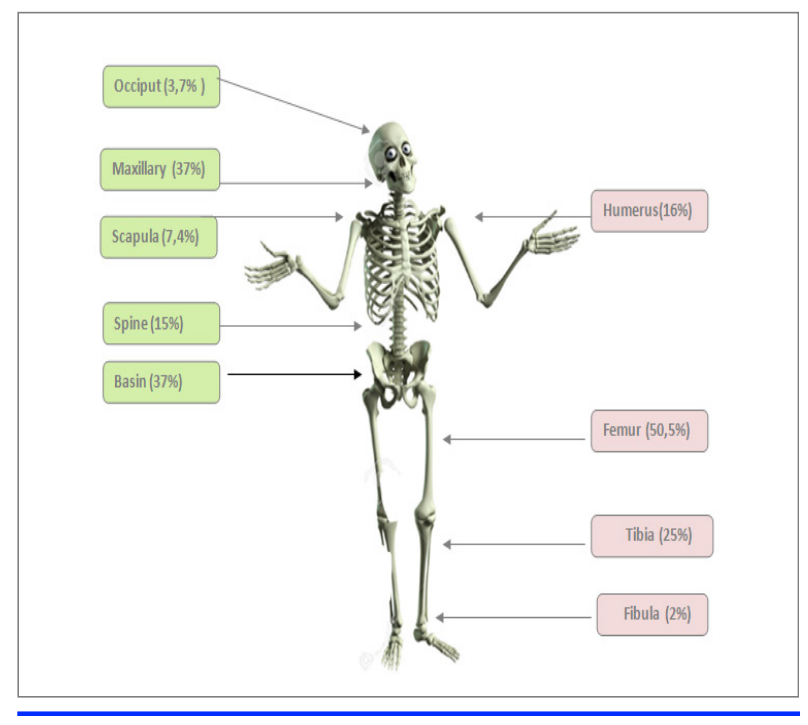

Figure b. Seat Distribution of Osteosarcoma

has declined over time from $60 \%$ between 1994 and 2004 to $55 \%$ between 2005 and 2016 .

\section{Consultation period}

was 3.4 months ( 15 days to 14 months). It was shorter in patients with an extra-axial seat tumor (3.1 months) compared to those with an axial seat tumor (5 months).

\section{Chief complaints}

The most common complaint was pain $(82 \%)$, swelling $(74 \%)$, trauma $(10 \%)$, fracture $(6.5 \%)$, and functional impairment (5.5\%).

\section{Physical signs}

The mean tumor size was $11 \mathrm{~cm}$ ( 6 to $18 \mathrm{~cm}$ ). The mean size observed between 1978 and 1993 was $14 \mathrm{~cm}$. There was a steady and gradual decrease in tumor size afterward to reach an average size of $11 \mathrm{~cm}$.

\section{Site distribution}

the majority of OS $(86,5 \%)$ arose in the appendicular skeleton. the lower limbs were the most affected (77, $5 \%)$. Most common was the femur $(50.5 \%)$ followed by tibia (25\%), humerus (16\%), ulna $(6.5 \%)$ and fibula $(2 \%)$. The axial skeleton was affected in $13.5 \%$ of cases pelvis $(37 \%)$, followed by maxillary bone $(37 \%)$, spine (15\%), scapula $(7.4 \%)$ and occiput $(3.7 \%)$. Nevertheless, the rate of patients with axial tumor location has increased over the years, from $7 \%$ between 1978 and 1993 to $22 \%$ from the year 2000 .

The preferred site was long bones in young patients and flat bones in elderly ones. In fact, the age-specific incidence rate for appendicular malignant osteosarcomas was lower among adults aged over 60 years $(25 \%)$ and was higher among adolescents aged 10-19 years (97\%). However, the age-specific incidence rate for axial malignant osteosarcoma was lower among children and adolescents aged less than 20 years $(2 \%)$ and higher among adults aged over 60 years $(71 \%)$. Moreover, differences in distribution by gender were also observed given that 


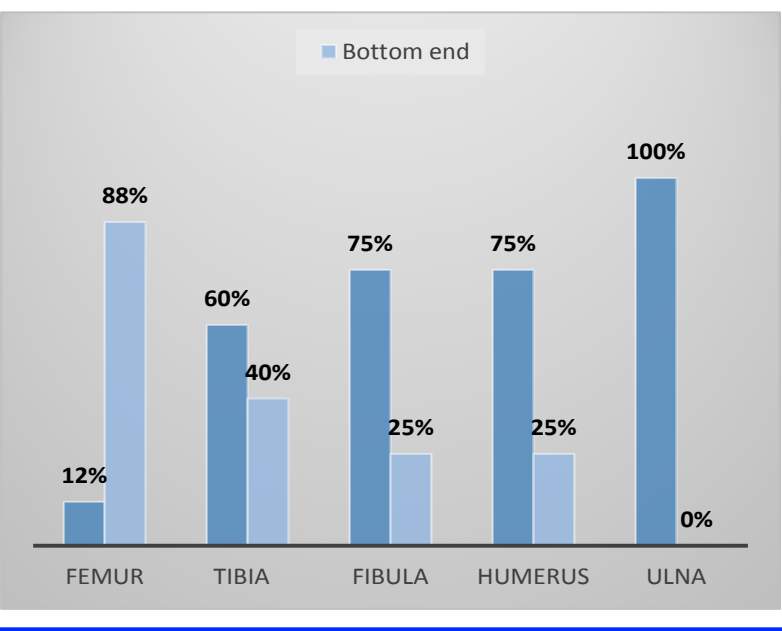

Figure c. Distribution of Osteosarcoma of Extra-axial Seat

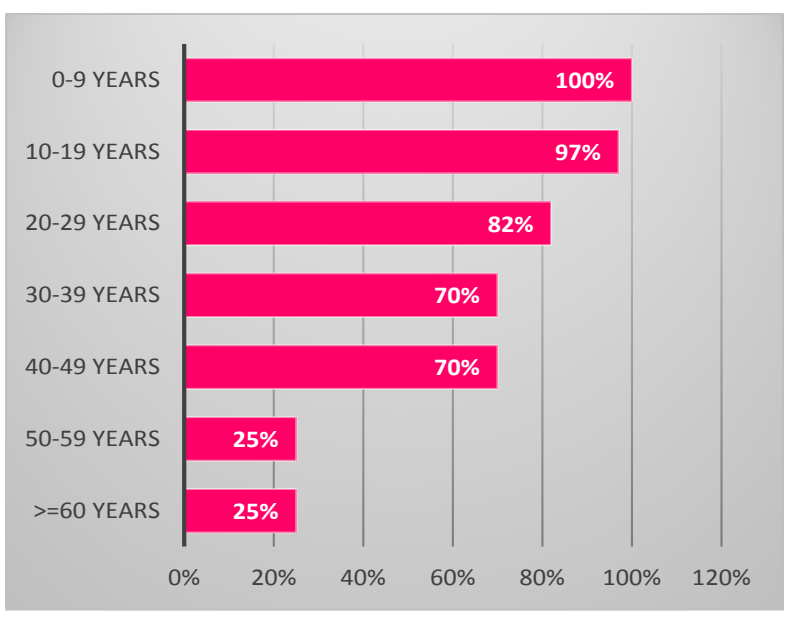

Figure e. Distribution of Extra Axial Seat According to Age

among axial malignant OS cases, the distribution was higher among males (19\%) compared with females (11\%) whereas, among appendicular malignant OS cases, the distribution was slightly higher among females $(88 \%)$ compared with males (84\%) (Figure 2A-2B).

\section{Radiological findings}

For local extension assessment, conventional radiography was done for all patients and MRI was done for $38.5 \%$ of patients. For distance extension assessment, chest CT was done for $72 \%$ of patients and bone scintigraphy for $67 \%$ of patients. The lytic radiological aspect was predominant (45\%). The condensing and mixed ones were $21 \%$ each. Of the total cases, $21 \%$ were found with metastases. Thus, localized osteosarcoma was more frequent than the metastatic form (79\% vs 21\%). Axial seat tumors were predominantly localized whereas extra-axial seat tumors were mostly metastatic. Metastases were mainly pulmonary (85\%) and bone (20\%).

\section{Histological findings}

The histological diagnosis was carried out in $95.5 \%$ of the patients on a piece of surgical biopsy and in $4.5 \%$ of

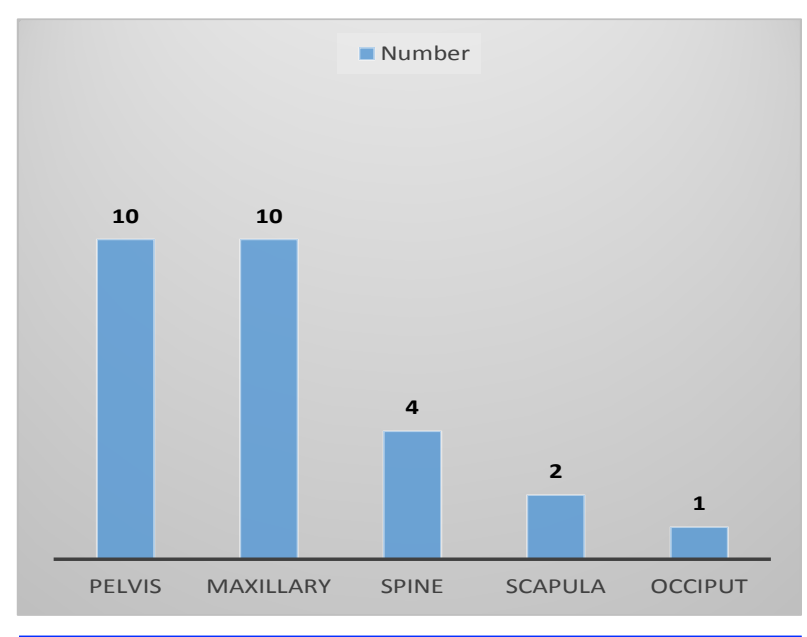

Figure d. Distribution of Osteosarcoma of Axial Seat

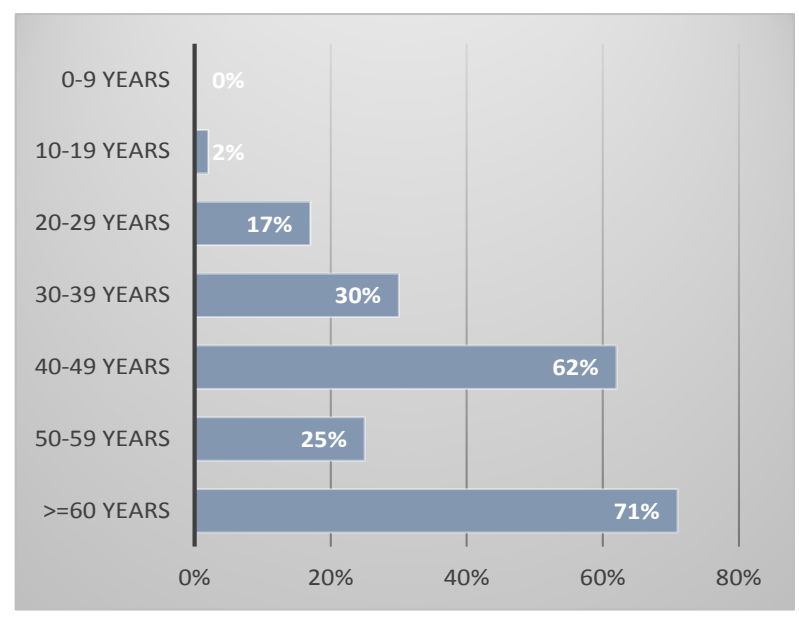

Figure f. Distribution of Axial Seat According to Age

the cases on a surgical specimen. The common form was the most frequent regardless of sex and location. The osteoblastic lesion was the most common OS variant (47\%) (Figure 4). Median progression-free survival was 17 months for the osteoblastic form, 20 months for the chondroblastic form and 6 months for the fibroblastic forms.

\section{Outcomes}

The overall survival of our population was 78 $\%$ at 5 years. Factors influencing overall survival at univariate analysis were: Trauma history $(\mathrm{p}=0.037)$, functional impotence and movement limitation $(\mathrm{p}=0.01)$, local treatment of the primary tumor $(p=0.008)$ and local treatment of distant metastases $(p=0.003)$. Progression-free survival (PFS), was $90.5 \%$ at 5 years.

\section{Discussion}

Our study is the first national report that describes the epidemiological characteristics of primary malignant osteosarcoma in Tunisia. 
Incidence

$\mathrm{OS}$ is a rare tumor with an estimated frequency between 0.5 and $2 \%$ of all cancers [8]. The incidence of OS during childhood and adolescence is estimated to be between 3 and 4.5 cases per million inhabitants per year [2-9]. A higher incidence has been reported in Italy, Latin America and some African countries, while lower occurrence rates have been observed in other countries such as Western Australia and the United States [2]. In our series, 200 cases were recorded over a period of 37 years with a mean incidence of 5 cases per year.

Age

According to Parkin. et al. [10] $80 \%$ of osteosarcomas occur in children and adolescents with an average age of 14 years. Outstanding cases have been reported before the age of 5 years. According to Philip et al. an age of fewer than 12 years is a predicting factor of poor prognosis [11]. In our study, the mean age at the time of diagnosis was 18 years. As a rule, the incidence rates of OS are age-related and have a bimodal distribution. The first well-defined peak occurs mainly during the second decade of life: between 15 and 19 years [2]. This peak is followed by a decrease in the incidence of occurrence with a plateau in subjects aged between 25 and 60 years. A second lower peak is observed in the 7 th and 8 th decades of life. The distribution of our patients throughout all ages failed to show this bimodal age distribution. We found only a single peak during adolescence and a plateau during adulthood. The lack of the second peak in our population can be related to the fact that we didn't include patients having Paget disease which occurs at an elderly age.

\section{Sexes}

In most series, men are more affected than women in each age group with a male-to-female ratio close to 1.7 [2]. These ratios are consistent with those of our series. Nevertheless, in some studies women are more affected than men in patients under the age of 15 years, as well as in elderly subjects especially those with a history of neoplasia [2-9]. These data suggest that bone growth as well as hormonal changes related to puberty may be involved in the occurrence of osteosarcomas [12].

-The timing of consultation is a decisive prognostic factor given the high malignancy potential of OS. In our study the average consultation period was 3.4 months and has reached 15 months in some cases. This delay can be explained by the lack of specificity of clinical signs given by the fact that swelling is often mistaken as a simple inflammation or an infection especially when the discovery is concurrent with a trauma. In other cases the low socio-economic level or the low level of education of some parents can be involved in the delay in therapeutic management.

The clinical semiology of OS is based on the triad Pain, swelling and functional impotence. The main clinical sign is pain present in 80 to $85 \%$ of cases in the majority of series $[13,14]$. In our study, which was the complaint in $82 \%$ of cases. It initially has a mechanical rhythm that gradually overlaps with an inflammatory type resulting in a total functional impotence of the patient [15-16]. Delphine et al. [17] showed that in one-third of cases swelling may be absent.

\section{Localization}

OS is mainly located in the peripheral skeleton and sites where bone growth is highest $[6,18]$. In our study, the most frequent localizations were the femur $(50.5 \%)$, the tibia (25\%) and the humerus (16\%). In Arndt [19], Campanacci and Widhe [20], in which 3433 OS cases were collected, knee infiltration accounted for more than half of cases $(53 \%)$ followed by humerus $(10 \%)$.

\section{Radiological findings}

\section{Local extension}

Most osteosarcomas occur in the metaphyseal region of bones which radiologically abnormalities of bone structures at a type of osteolysis and osteocondensation.

In our study, standard radiography of the affected bone was performed in all our patients. It showed osteolysis images in $45 \%$ of cases, osteocondensating lesions in $21 \%$ of cases, and mixed lesions in $21 \%$ of cases.

Magnetic resonance imaging Is an essential tool for determining intra-osseous tumor extension and soft tissue involvement. It is also a good tool for showing skip metastases [21].

In our study, MRI indicated soft tissue invasion in $49.5 \%$ of cases, joint invasion in $23 \%$ of cases and vascular and nerve infiltration in $16 \%$ of cases. Skips metastases were noted in only $6 \%$ of cases.

\section{Distance extension}

The assessment and the prognosis of OS are dominated by the occurrence of metastases. These metastases appear mainly in the lungs. The sensitivity of computed tomography is higher than that of standard radiographs for the detection of lung metastases (88\% vs. 57\%) [22].

Diagnostic criteria have been defined by the "European and American Osteosarcoma Study Group 1 EURAMOS" [23].

Bone scintigraphy is indicated before any clinical or radiological suspicion of malignant bone pathology (search for tumor and skip metastases as well as exploration of the whole body) [24-25].

\section{-Prognostic factors}

\section{-Pulmonary metastases}

The presence of visible pulmonary metastases at the first assessment is the main prognostic factor. Without treatment, survival from their diagnosis is between 6 and 10 months. In our study pulmonary metastases were associated with poor prognosis.

\section{- Tumor size}

We didn't found any correlation between tumor size and survival in our population. However, a large tumor 
contains many more cells and presents a greater risk than a clone cell which is resistant to chemotherapy. Large tumors are thus generally more chemoresistant than the small ones, and under these conditions, they remain a poor prognosis.

\section{-Tumor site}

Tumor location remains a major prognostic factor because the axial locations are usually large volume and their local treatment is difficult. In the Mac Kenna series [25], 5-year survival of central osteosarcomas did not exceed 5\%. In most series, survival expectancy after hip disarticulation for lower femur osteosarcoma was between 15 and $25 \%$ whereas it reached 25 to $40 \%$ after amputation of the leg for upper tibia sarcoma and more than $50 \%$ for more distal tumors. Overall, the best survival rate is obtained for proximal humeral osteosarcomas (more than $80 \%$ survival) then the tibia locations proximal ( $80 \%$ survival) and finally distal and proximal femur respectively with $73 \%$ and $70 \%$ survival. In our population, overall survival of femur location was $61.5 \%$ at 5 years

\section{-Histological findings}

The incidence of histologic forms seems to be variable from one series to another [27-28]. Osteoblastic forms would have the worst prognosis with a lower recurrence-free survival at 5 years. In Houston study of MD Anderson, the recurrence-free survival was $57 \%$ for the osteoblastic form versus $80 \%$ for the fibroblastic one. In Memorial Hospital, the recurrence-free survival at 5 years was $67 \%$ for osteoblastic and chondroblastic forms and $93 \%$ for fibroblastic forms. In the Dahlin series, there was no difference in prognosis between the telangiectatic and conventional form. The telangiectatic form has a very variable prognosis according to the series since their prognosis is better in the Raymond and Jaffee series (recurrence-free survival at 5 years of $40 \%$ ). In our Data, median progression-free survival was 17 months for the osteoblastic form, 20 months for the chondroblastic form and 6 months for the fibroblastic forms.

- The histological response to neoadjuvant chemotherapy is currently one of the prognostic factors for major osteosarcomas. The 5-year survival of good responders ranges from 60 to $80 \%$ versus 40 to $60 \%$ in the case of a poor histological response [29-30].

Other prognostic factors are currently under evaluation [31].

- BMP (Bone Morphogenetic Protein): When present, tumors are resistant to chemotherapy and $83 \%$ of cases are metastatic to the lungs.

- Quantification of (dDNA) P glycoprotein detected by immunohistochemistry, which evaluates the predictable response to chemotherapy,

- The presence of the mdrl gene also seems to have a value in the prediction resistance to chemotherapy.

In conclusion, this study is the first large national population-based incidence report of osteosarcoma in Tunisia. It provides a better understanding of the epidemiologic characteristics of this disease.

\section{Acknowledgements}

We appreciate the cooperation of Pathology and Radiotherapy service of the Salah Aziaez Institute.

\section{References}

1. American cancer society [internet]. Available from: http:// www.cancer.org/cancer/bonecancer/detailedguid/bonecancerkey- statistics

2. Mirabello L, Troisi RJ, Savage SA (2009). International osteosarcoma incidence patterns in children and adolescents, middle ages, and elderly persons. Int J Cancer, 125, 229-34.

3. Gentet JC, Bouvier C, Pillon P, Jouve JL, Lautraite C, Coze C et al. Ostéosarcomes. Med Ther Ped. 2002; 3 (5): 169-173. PubMed | Google Scholar

4. Hayden JB, Hoang BH. Osteosarcoma: basic science and clinical implications. Orthop Clin North Am. 2006;37(1):1-7.

5. Bramer JAM, Somford MP. The epidemiology of primary skeletal malignancy. Orthopaedics and trauma 2010;24(4);247-51.

6. Lowry RB. The case for reviving the birth-defects database. The Vancouver Sun. May 15, 2012:Opinion.

7. Lowry RB, Thunem NY, Anderson-Redick S. Alberta Congenital Anomalies Surveillance System: 1980-1986. Can Med Assoc J. 2007;141:1155-1159.

8. Madewell JE, Ragsdale BD, Sweet: Radiologic and pathologic analysis of solitary bone lesions. Part I : internal margins. Radiol. Clin. North Am. $1981 ; 19$ : 715-748

9. C.A.Stiller,S.S. Bielack,G.Jund et al,"Bone tumours in European children and adolescents, 1978-1997. Report from the Automated Childhood CancerInformationSystemproject ,'European Journal of Cancer2006; 14 : 2124-2135

10. Parkin, D. M., Stiller, C. A., \& Nectoux, J. (1993). International variations in the incidence of childhood bone tumours. International journal of cancer, 53(3), 371-376.

11. Bergman, P. J., MacEwen, E. G., Kurzman, I. D., Henry, C. J., Hammer, A. S., Knapp, D. W., ... \& Norris, A. M. (1996). Amputation and carboplatin for treatment of dogs with osteosarcoma: 48 cases (1991 to 1993). Journal of Veterinary Internal Medicine, 10(2), 76-81.

12. S.Savage; ;.Mirabello :Using Epidemiology and Genomics to Understand Osteosarcoma Etiology .Hindawi Publishing Corporation Sarcoma 2011,ArticleID548151 13 pages

13. Kalifa C : La chimiothérapie de l'ostéosarcome, tumeur maligne primitives 1990

14. Widhe B : Symptomes initiaux et dispositifs cliniques dans le sarcome d'Ewing et l'ostéosarcome, The journal of Bone and Joint Surgery $200082: 667$

15. Andon A: Les tumeurs osseuses Communication de l'institut Gustave- Roussy février 2004

16. Dubousset J.F., Missenard G., Kalifa C. 1991. Management of osteogenic sarcoma in children and adolescent. Clin Orthop 270: 52-59.

17. Delepine G, Goutallier D. : La resection dans le traitement des ostéosarcomes des membres. L'amputation conservera-t-elle des indications ? revue de chirurgie orthopedique.1985,71,451-461

18. R. Eyre, R. Feltbower, P. W. James et al., "The epidemiology of bone cancer in 0-39 year olds in northern England, 1981-2002,"BMC Cancer, vol. 10, article357, 2010.

19. J. Blackwell, T. Threlfall, and K. A. McCaul, "Primary malignant bone tumours in Western Australia, 1972-1996," Pathology,2005;37: 278- 283

20. T.A.Damron,W.G.Ward,A.Stewart,"Osteosarcoma, chondrosarcoma, and Ewing's sarcoma: national cancer 
data base report," Clinical Orthopaedics and Related Research.2007;459: 40-47

21. N. El Ghazouli, W. Zamiati, S. Lezar, R. Kadiri: Apport de l'imagerie dans l'ostéosarcome Service Central de Radiologie, CHU Ibn Rochd, Casablanca, Maroc Rev Maroc Chir Orthop Traumato $2006 ; 28: 25-29$

22. Vanel, D., Henry-Amar, M., Lumbroso, J., Lemalet, E., Couanet, D., Piekarski, J. D., ... \& Le Chevalier, T. (1984). Pulmonary evaluation of patients with osteosarcoma: roles of standard radiography, tomography, CT, scintigraphy, and tomoscintigraphy. American journal of roentgenology, 143(3), 519-523.

23. Whelan, J. S., et al. "EURAMOS-1, an international randomised study for osteosarcoma: results from prerandomisation treatment." Annals of Oncology 26.2 (2014): 407-414.

24. Thierry P ; Jean-Yves B ; Christian C: Standards, options et recommandations pour le diagnostic, le traitement et la surveillance de l'ostéosarcome Bulletin du cancer (Paris); 1999 ; vol 86 ; 159-176

25. Mc Kenna, Robert J., Charles P. Schwinn, and Norman L. Higinbotham. "Osteogenic sarcoma in children." California medicine 103.3 (1965): 165.

26. Pratt, Charles B., J. E. Champion, I. D. Fleming, B. Rao, A. P. M. Kumar, W. E. Evans, A. E. Green, and S. George. "Adjuvant chemotherapy for osteosarcoma of the extremity." Cancer 65 (1990): 439-445.

27. C. Fletcher,K. Unni,F. Mertens: Pathology and Genetics of Tumours of Soft Tissue and Bone. World Health Organization Classification of Tumours 2002

28. Dahlin D C, Cowentry : Ostogenie sarcoma : a study of 600 cases ; J Bone Joint Surgeon 1977,49A 308-31

29. Davis A, Belle R, Goodwin P: Prognostic factors in osteosarcoma : acritical review. J Clin Oncol 1994 ; 12 : 423-

30. Saeter G, Elomaa I, Wahlqvist Y, Alvegard TA, Wiebe T, Monge O, et al.: Prognostic factors in bone sarcomas. Acta Orthop Scand Suppl $1997 ; 273: 156-60$

31. Tomeno B ; Forest M: Interet pronostique des données histologiques de la classification de Ennecking ("staging et grading") dans les tumeurs primitives de l'appareil locomoteur. Conférences d'enseignement de la Sofocot $1994 ; 46$ : 183-193.

\section{(c) (i) (8)}

This work is licensed under a Creative Commons AttributionNon Commercial 4.0 International License. 